\title{
Induced superconductivity in the quantum spin Hall edge
}

\author{
Sean Hart ${ }^{1 \dagger}$, Hechen Ren ${ }^{1 \dagger}$, Timo Wagner ${ }^{1}$, Philipp Leubner ${ }^{2}$, Mathias Mühlbauer ${ }^{2}$, Christoph Brüne ${ }^{2}$, \\ Hartmut Buhmann ${ }^{2}$, Laurens W. Molenkamp ${ }^{2}$ and Amir Yacoby ${ }^{1 \star}$
}

Topological insulators are a newly discovered phase of matter characterized by gapped bulk states surrounded by conducting boundary states ${ }^{1-3}$. Since their theoretical discovery, these materials have encouraged intense efforts to study their properties and capabilities. Among the most striking results of this activity are proposals to engineer a new variety of superconductor at the surfaces of topological insulators ${ }^{4,5}$. These topological superconductors would be capable of supporting localized Majorana fermions, particles whose braiding properties have been proposed as the basis of a fault-tolerant quantum computer ${ }^{6}$. Despite the clear theoretical motivation, a conclusive realization of topological superconductivity remains an outstanding experimental goal. Here we present measurements of superconductivity induced in two-dimensional $\mathrm{HgTe} / \mathrm{HgCdTe}$ quantum wells, a material that becomes a quantum spin Hall insulator when the well width exceeds $d_{c}=6.3 \mathrm{~nm}$ (ref. 7). In wells that are $7.5 \mathrm{~nm}$ wide, we find that supercurrents are confined to the one-dimensional sample edges as the bulk density is depleted. However, when the well width is decreased to $4.5 \mathrm{~nm}$ the edge supercurrents cannot be distinguished from those in the bulk. Our results provide evidence for supercurrents induced in the helical edges of the quantum spin Hall effect, establishing this system as a promising avenue towards topological superconductivity. In addition to directly confirming the existence of the topological edge channels, our results also provide a measurement of their widths, which range from $180 \mathrm{~nm}$ to $408 \mathrm{~nm}$.

Topological superconductors, like topological insulators, possess a bulk energy gap and gapless surface states. In a topological superconductor, the surface states are predicted to manifest as zero-energy Majorana fermions, fractionalized modes that pair to form conventional fermions. Owing to their non-Abelian braiding statistics, achieving control of these Majorana modes is desirable both fundamentally and for applications to quantum information processing. Proposals towards realizing Majorana fermions have focused on their emergence within fractional quantum Hall states ${ }^{8}$ and spinless $p+i p$ superconductors ${ }^{9}$, and on their direct engineering using $s$-wave superconductors combined with topological insulators or semiconductors ${ }^{10,11}$. Particularly appealing are implementations in one-dimensional (1D) systems, where restriction to a single spin degree of freedom combined with proximity to an $s$-wave superconductor would provide the basis for topological superconductivity ${ }^{12}$. Effort in this direction has been advanced by studies of nanowire systems ${ }^{13-18}$ and by excess current measurements on InAs/GaSb devices ${ }^{19}$.
An attractive route towards a $1 \mathrm{D}$ topological superconductor originates from the $2 \mathrm{D}$ quantum spin Hall (QSH) insulator. This topological phase of matter was recently predicted ${ }^{20,21}$ and observed $^{22,23}$ in transport measurements of $\mathrm{HgTe} / \mathrm{HgCdTe}$ quantum wells thicker than a critical thickness $d_{\mathrm{C}}=6.3 \mathrm{~nm}$. Due to strong spin-orbit coupling the bulk bands of the system invert, crossing only at the edges of the system to form 1D counterpropagating helical modes. Time-reversal symmetry ensures protection of these modes against elastic backscattering over distances shorter than the coherence length ${ }^{24}$. The helical nature of the edge modes makes them a particularly appealing path towards topological superconductivity, owing to the intrinsic elimination of their spin degree of freedom. Here we report measurements of supercurrents induced in $\mathrm{HgTe} / \mathrm{HgCdTe}$ quantum well heterostructures. As the system enters the QSH regime we find that these supercurrents become confined to the topological edge modes, verifying their existence and providing a microscopic picture of the QSH state.

Our approach consists of a two-terminal Josephson junction, with a rectangular section of quantum well located between two superconducting leads (Fig. 1). At a given bulk carrier density, the presence or absence of helical edge channels influences the supercurrent density profile across the width of the junction. In the simplest case the supercurrent density is uniform throughout the device, and edge channels are indistinguishable from bulk channels (Fig. 1a). This behaviour would be expected for a non-topological junction (quantum well width smaller than $d_{\mathrm{C}}$ ), or in a topological junction (quantum well width larger than $d_{\mathrm{C}}$ ) far from the bulk insulating regime.

In a topological junction, decreasing the bulk carrier density brings the device closer to the QSH insulator regime (Fig. 1b). Scanning superconducting quantum interference device (SQUID) measurements suggest that over a range of bulk densities the QSH edge channels coexist with bulk states, and can carry considerably more edge current than would be expected for a non-topological conductor $^{25}$. In the two-terminal configuration, this helical edge contribution appears as peaks in the supercurrent density at each edge. When the bulk density becomes sufficiently low, these edge peaks are the only features in the supercurrent density (Fig. 1c). Then the supercurrent is carried solely along the helical edges, and the system is in the regime of the quantum spin Hall superconductor.

Placing such a Josephson junction in a perpendicular magnetic field $B$ provides a way to measure the supercurrent density in the quantum well. In general, the maximum supercurrent that can flow through a Josephson junction is periodically modulated by a

${ }^{1}$ Department of Physics, Harvard University, Cambridge, Massachusetts 02138, USA, ${ }^{2}$ Physikalisches Institut (EP3), Universität Würzburg, 97074

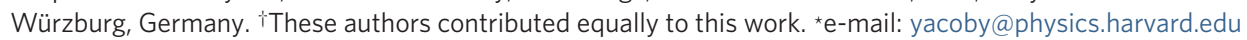


a
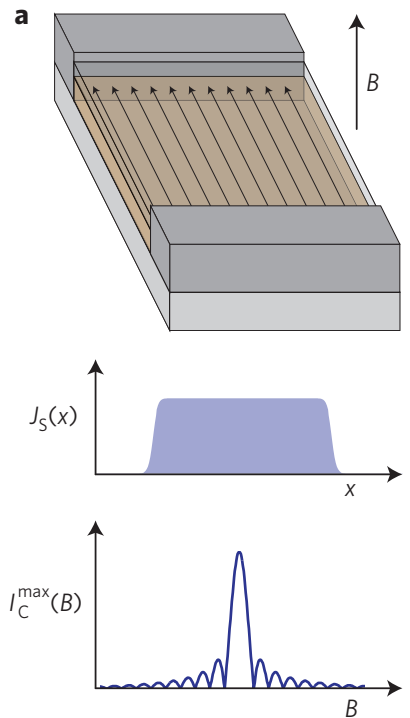

b
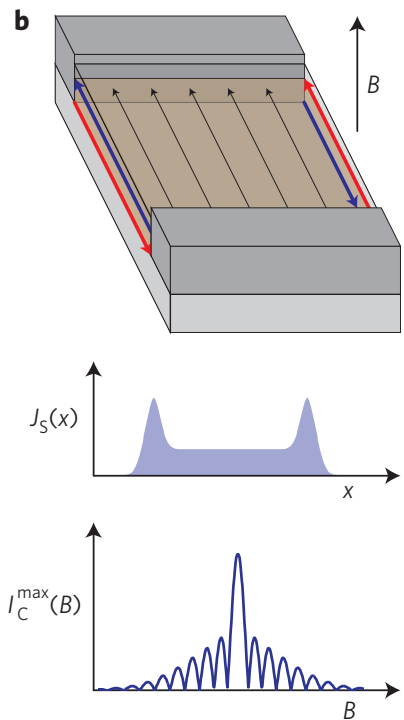
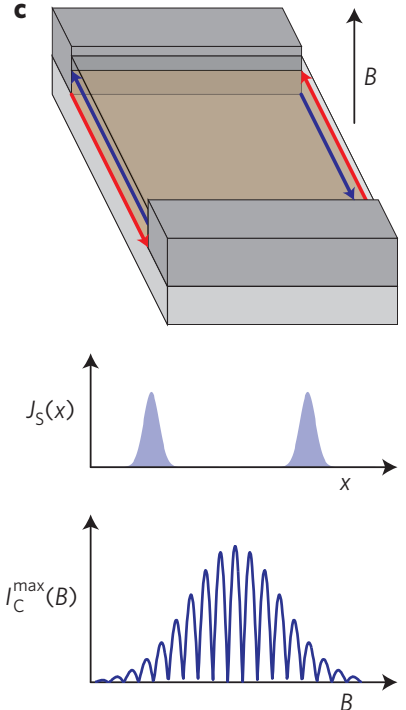

Figure 1 | Expected two-terminal behaviour in different regimes of a topological quantum well. a, When the bulk of a sample is filled with charge carriers, supercurrent can flow uniformly across the junction, corresponding to a flat supercurrent density $J_{S}(x)$. A perpendicular magnetic field $B$ modulates the maximum critical current $I_{C}^{\max }$, resulting in a single-slit Fraunhofer interference pattern. $\mathbf{b}$, As the bulk carriers are depleted, the supercurrent density develops peaks owing to the presence of the helical edges. This evolution towards edge-dominated transport appears in the interference pattern as a

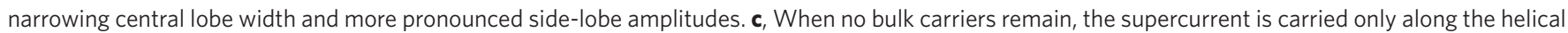
edges. In this regime the interference results in a sinusoidal double-slit pattern, with an overall decay in $B$ that is determined by the width of the edge channels.

a

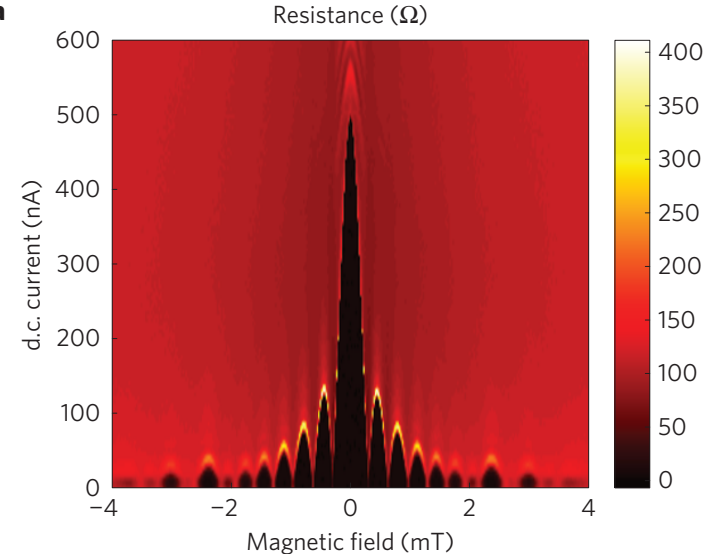

c

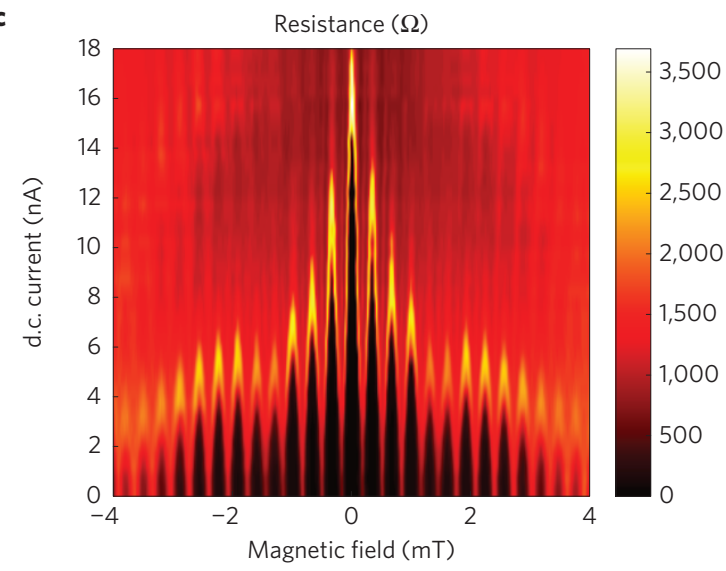

b

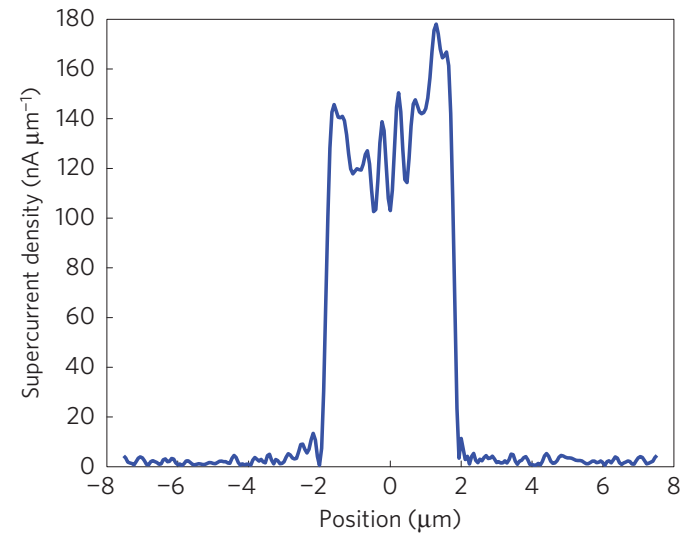

d

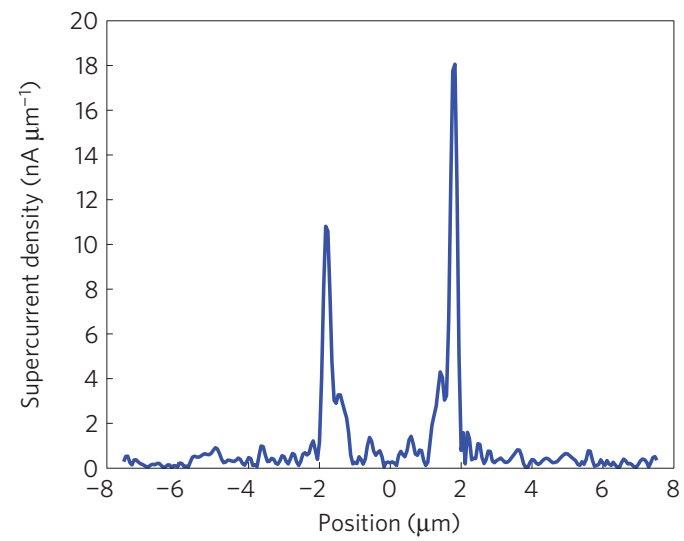

Figure 2 | General behaviour observed in the topological Josephson junction. a, A map of the differential resistance across the junction, measured with the top gate at $V_{G}=1.05 \mathrm{~V}$, shows the single-slit interference characteristic of a uniform supercurrent density. $\mathbf{b}$, The supercurrent density, extracted for $V_{G}=1.05 \mathrm{~V}$, is consistent with trivial charge transport throughout the bulk of the junction. $\mathbf{c}$, When the top-gate voltage is lowered to $V_{G}=-0.425 V_{\text {, the }}$ differential resistance shows a more sinusoidal interference pattern. $\mathbf{d}$, Using the interference envelope measured at $V_{\mathrm{G}}=-0.425 \mathrm{~V}$, the supercurrent density is clearly dominated by the contribution from the edges. In this regime almost no supercurrent passes through the bulk. 

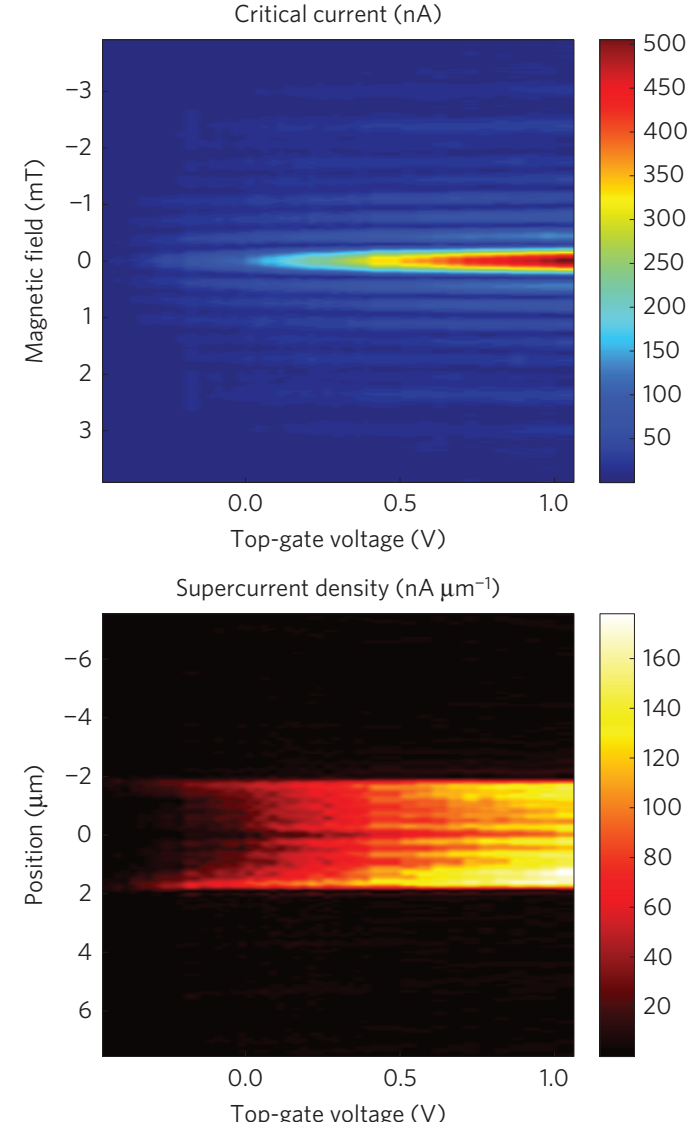

e

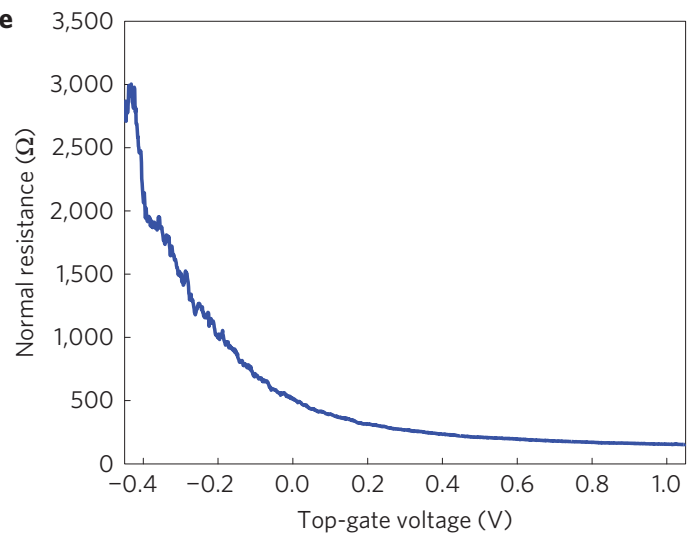

b

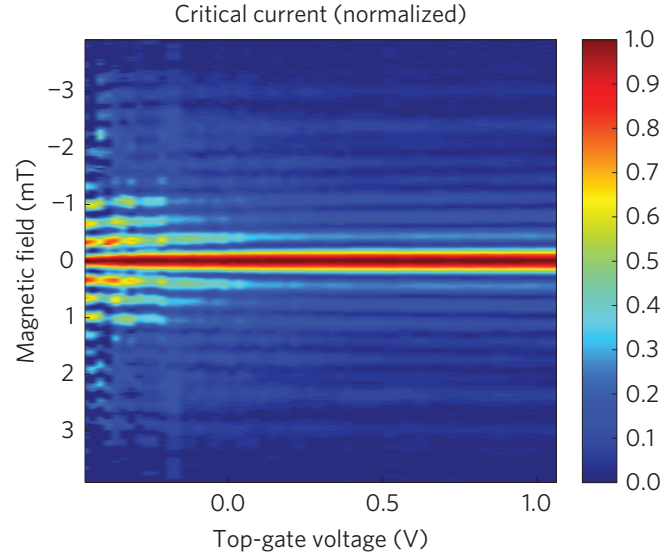

d
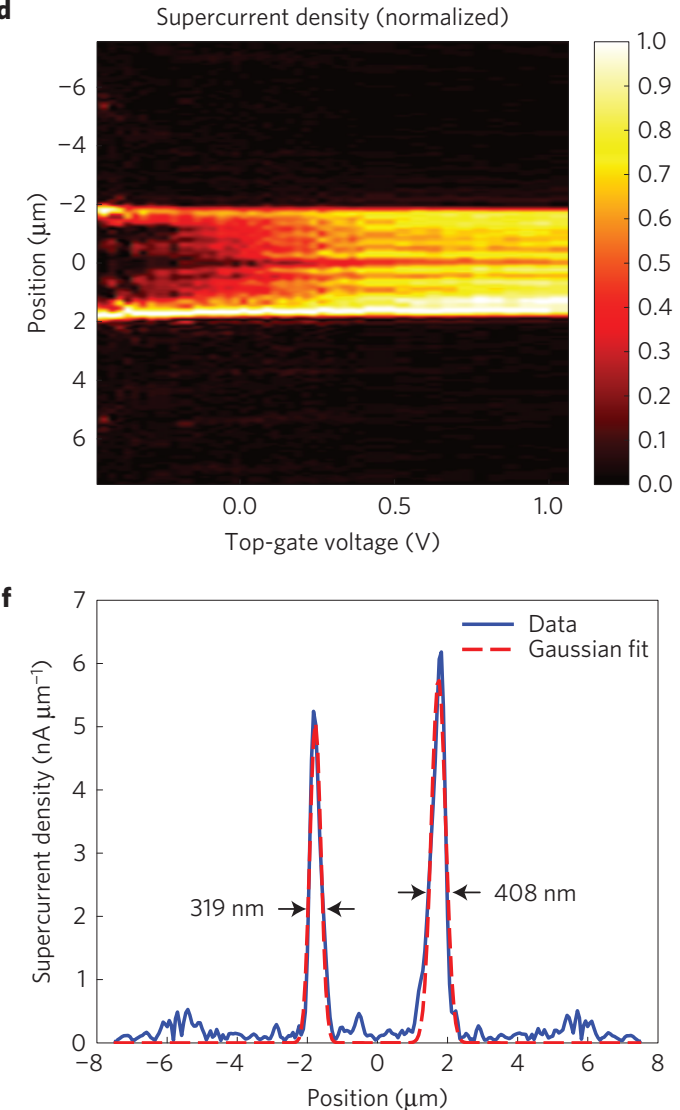

Figure 3 | Continuous evolution with gating in the topological Josephson junction. $\mathbf{a}$, As the top gate is varied from $V_{\mathrm{G}}=1.05 \mathrm{~V}$ to $V_{\mathrm{G}}=-0.45 \mathrm{~V}$, the maximum critical current decreases from $505 \mathrm{nA}$ to $5.7 \mathrm{nA}$. b. Normalizing the interference patterns to their peak values reveals the evolution towards sinusoidal interference. c, Using the envelope at each gate voltage, the evolution of the supercurrent density can be visualized. $\mathbf{d}$, By normalizing each supercurrent density to its maximum value, the transition from trivial to edge-dominated supercurrent transport can be clearly seen. $\mathbf{e}$, This transition occurs as the normal device resistance increases from $160 \Omega$ to 3,000 $\Omega$. f, At the most negative gate voltage, $V_{G}=-0.45 \mathrm{~V}$, the supercurrent density provides a measurement of the edge widths.

magnetic field. Typically, the period of the modulation corresponds to the magnetic flux quantum $\Phi_{0}=h / 2 e$. In our junctions this period matches the area of the HgTe region plus half the area occupied by each contact, a result of the Meissner effect. The particular shape of the critical current interference pattern depends on the phase-sensitive summation of the supercurrents traversing the junction ${ }^{26}$. In the case of a symmetric supercurrent distribution, this integral takes the simple form:

$$
I_{\mathrm{C}}^{\max }(B)=\left|\int_{-\infty}^{\infty} \mathrm{d} x J_{\mathrm{S}}(x) \cos \left(2 \pi L_{\mathrm{J}} B x / \Phi_{0}\right)\right|
$$

Here $L_{\mathrm{J}}$ is the length of the junction along the direction of current, accounting for the magnetic flux focusing from the contacts.

It is evident that different supercurrent densities $J_{S}(x)$ in the junction can give rise to different interference patterns $I_{\mathrm{C}}^{\max }(B)$. The flat supercurrent density of a trivial conductor corresponds to a single-slit Fraunhofer pattern $\left|\left(\sin \left(\pi L_{\mathrm{J}} B W / \Phi_{0}\right)\right) /\left(\pi L_{\mathrm{J}} B W / \Phi_{0}\right)\right|$, characterized by a central lobe width of $2 \Phi_{0}$ and side lobes decaying with $1 / B$ dependence (Fig. 1a). As helical edges emerge, this single-slit interference evolves towards the more sinusoidal oscillation characteristic of a SQUID (Fig. 1b). The central lobe 
a

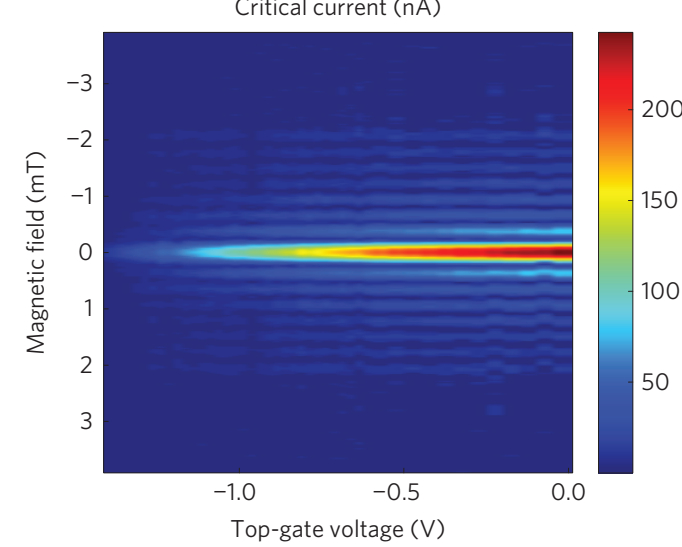

c

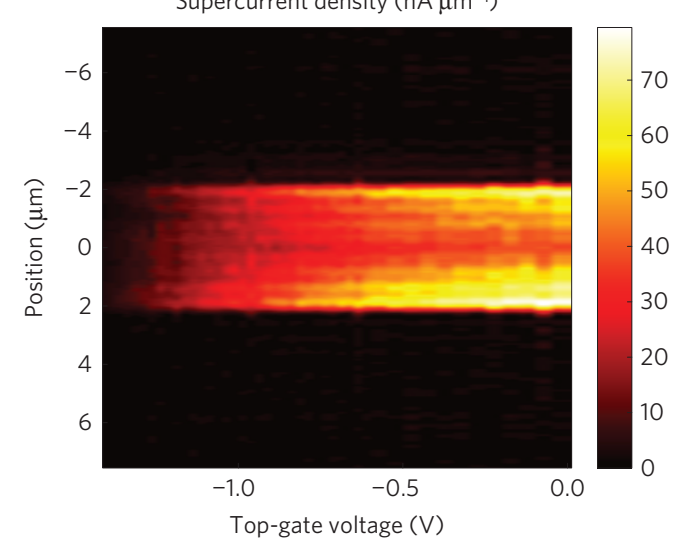

e

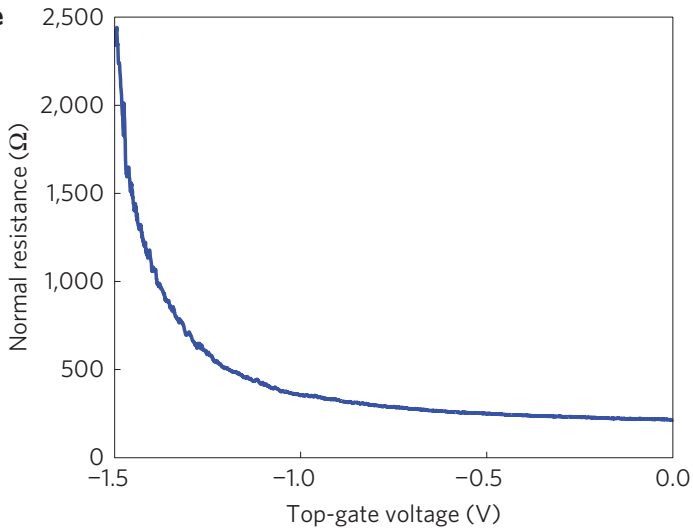

b

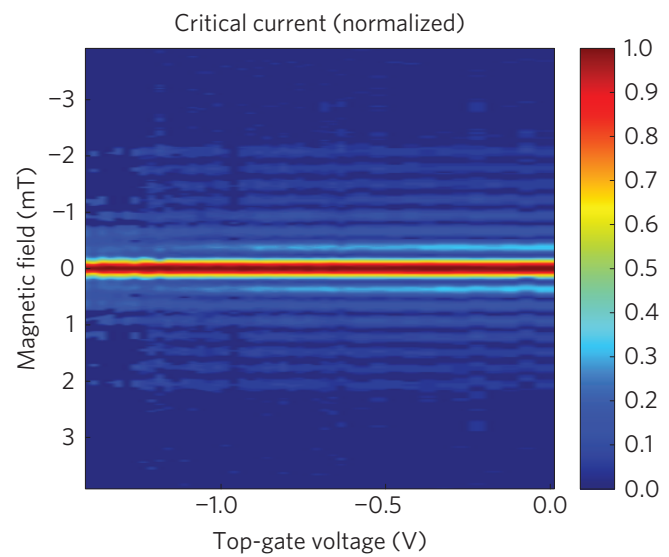

d

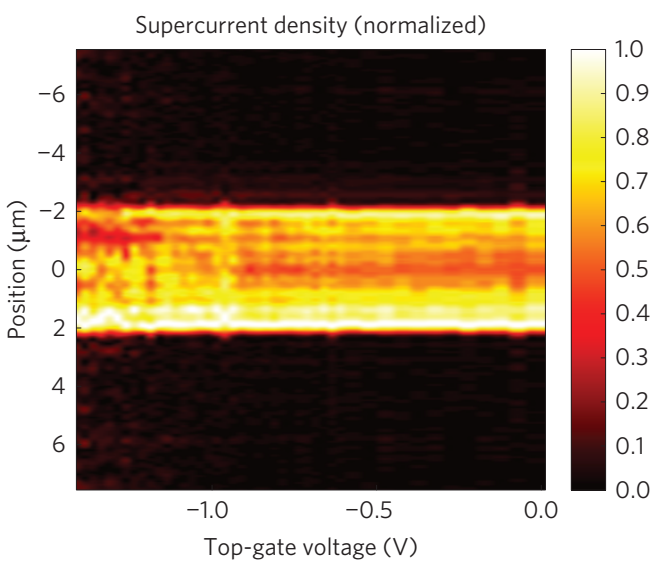

f

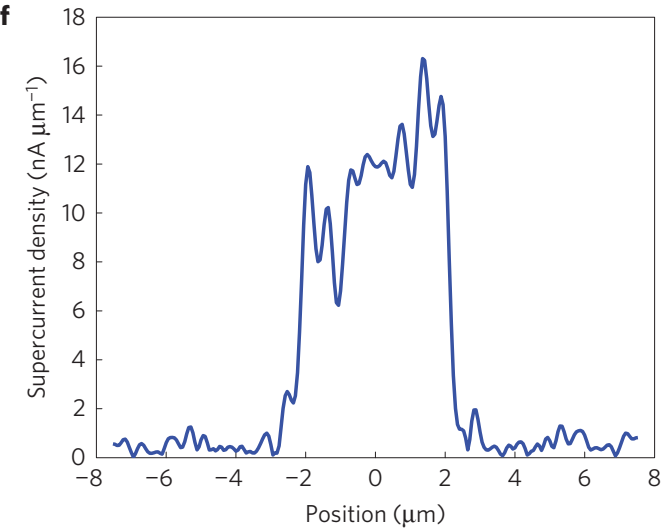

Figure 4 | Continuous evolution with gating in the non-topological Josephson junction. a, As the top gate is varied from $V_{G}=0 \vee$ to $V_{G}=-1.5 V$, the maximum critical current decreases from $243 \mathrm{nA}$ to $4.4 \mathrm{nA}$. b. Normalizing the interference patterns to their peak values shows the stability of the single-slit pattern over a wide range of gating. $\mathbf{c}$, Using the envelope at each gate voltage, the evolution of the supercurrent density can be visualized. d, Normalizing each supercurrent density to its maximum value shows that the supercurrent remains distributed throughout the device. $\mathbf{e}$, This roughly uniform supercurrent distribution remains even as the device resistance increases from $215 \Omega$ to almost 2,500 $\Omega$. f, A line trace of the supercurrent density close to depletion further demonstrates that the supercurrent flows throughout the device.

width shrinks to $\Phi_{0}$ when only edge supercurrents remain, with the side lobe decay determined by the widths of the edge channels (Fig. 1c). Measuring the dependence of $I_{\mathrm{C}}^{\max }$ on $B$ therefore provides a convenient way to measure the distribution of supercurrent in a junction. To quantitatively extract $J_{S}(x)$ from the measured quantity $I_{\mathrm{C}}^{\max }(B)$ we follow an approach where non-zero $I_{\mathrm{C}}^{\max }(B)$ minima are ascribed to an asymmetric supercurrent distribution ${ }^{27}$. Although other effects may lead to non-zero minima in $I_{\mathrm{C}}^{\max }(B)$, we consider here only the possibility of an odd component in $J_{S}(x)$. Full details of the extraction procedure can be found in the Supplementary Information.
To study how supercurrents flow in the QSH regime, we measure a Josephson junction consisting of a 7.5-nm-wide quantum well contacted by titanium/aluminium leads ${ }^{28}$. Our contact lengths are each $1 \mu \mathrm{m}$, and the contact separation is $800 \mathrm{~nm}$. The junction width of $4 \mu \mathrm{m}$ is defined by etched mesa edges. A voltage $V_{\mathrm{G}}$ applied to a global top gate allows us to tune the carrier density in the junction. At each value of $V_{\mathrm{G}}$ and $B$, the critical current $I_{\mathrm{C}}^{\max }$ is determined by increasing the current through the junction while monitoring the voltage across the leads. The behaviour observed in this device is reproducible in several other similar junctions, as reported in the Supplementary Information. 
As a function of the top-gate voltage, the overall behaviour of the junction evolves between two extremes. At more positive gate voltage and higher bulk density, the critical current envelope strongly resembles a single-slit pattern (Fig. 2a). This type of interference suggests a nearly uniform supercurrent density throughout the sample, confirmed by transformation to the $J_{s}(x)$ picture (Fig. 2b). This nearly flat distribution indicates that the quantum well is in the high carrier density regime of an essentially trivial conductor.

At more negative gate voltage and lower bulk density, the critical current envelope becomes close to a sinusoidal oscillation (Fig. 2c). The shift towards a SQUID-like interference pattern corresponds to the development of sharp peaks in supercurrent density at the mesa edges (Fig. 2d).

We can track this evolution in a single device by measuring the critical current envelope at a series of gate voltages. As the top gate is varied from $V_{\mathrm{G}}=1.05 \mathrm{~V}$ to $V_{\mathrm{G}}=-0.45 \mathrm{~V}$, the maximum critical current decreases from $505 \mathrm{nA}$ to $5.7 \mathrm{nA}$. At the same time, the overall critical current behaviour shows a narrowing of the central interference lobe, from $2 \Phi_{0}$ at positive gate voltages to $\Phi_{0}$ at negative gate voltages (Fig. 3a,b). The side lobes additionally become continuously more pronounced, indicating the confinement of supercurrent to channels at the edges of the junction (Fig. 3c,d). The normal resistance, measured at large bias to overcome superconductivity, increases from $160 \Omega$ to $\sim 3,000 \Omega$ over the range of this transition (Fig. 3e). Although it is possible to gate further towards depletion, the critical currents become too small to reliably measure and no meaningful supercurrent density can be extracted.

At the most negative gate voltage, $V_{\mathrm{G}}=-0.45 \mathrm{~V}$, we can estimate the widths of the supercurrent-carrying edge channels using a Gaussian line shape (Fig. 3f). Using this method, we find widths of $408 \mathrm{~nm}$ and $319 \mathrm{~nm}$ for the two edges. Our measurements of edge widths in another device with similar dimensions, as well as one with a $2 \mu \mathrm{m}$ mesa width, show edges as narrow as $180 \mathrm{~nm}$ (Supplementary Information). These width variations, as well as the normal-state resistance that is low compared with the resistance $h / 2 e^{2}$ for two ballistic $1 \mathrm{D}$ channels, suggest the presence of additional edge modes or of bulk modes coupled too weakly across the junction to carry supercurrent.

To provide further evidence that the observed edge supercurrents are topological in nature, we next turn to a heterostructure with a quantum well width of $4.5 \mathrm{~nm}$. In this device, the well width is smaller than the critical width $d_{C}$, so that the sample is not expected to enter the QSH regime. Near zero top-gate voltage and a normal resistance of $270 \Omega$, the critical current interference pattern has a maximum of $243 \mathrm{nA}$ and resembles a single-slit envelope (Fig. 4a,b). On energizing the top gate and decreasing the bulk density, the single-slit pattern persists. In contrast to the wide well sample, this behaviour corresponds to a supercurrent density that remains distributed throughout the junction even as the normal resistance rises to several $\mathrm{k} \Omega$ (Fig. $4 \mathrm{c}-\mathrm{f}$ ). As the edge supercurrents are present only when the well width is larger than $d_{\mathrm{C}}$, we conclude that our observations provide evidence for induced superconductivity in the helical QSH edge states.

By studying Fraunhofer interference, our measurements provide detailed information about the supercurrent distribution in $\mathrm{HgTe}$ quantum wells. In the quantum spin Hall regime, this interferometry confirms the existence of topological edge channels associated with the quantum spin Hall insulating state. Our observed supercurrent distributions additionally provide the first direct measurements of the helical edges' spatial extent. In general, our application of this Fourier imaging technique to HgTe quantum wells could be widely adopted as a method to elucidate the microscopic structure of topological materials. In our devices, the observation of Josephson supercurrents through the helical edge channels establishes the $\mathrm{HgTe} / \mathrm{HgCdTe}$ system as a platform in which to pursue topological superconductivity and Majorana bound states, whether through following existing theoretical proposals or those yet to be formulated ${ }^{29-31}$

Received 1 November 2013; accepted 17 June 2014;

published online 3 August 2014

\section{References}

1. Hasan, M. Z. \& Kane, C. L. Colloquium: Topological insulators. Rev. Mod. Phys. 82, 3045-3067 (2010)

2. Qi, X-L. \& Zhang, S-C. Topological insulators and superconductors. Rev. Mod. Phys. 83, 1057-1110 (2011).

3. Kane, C. L. \& Mele, E. J. Z2 topological order and the quantum spin Hall effect. Phys. Rev. Lett. 95, 146802 (2005).

4. Fu, L. \& Kane, C. L. Superconducting proximity effect and majorana fermions at the surface of a topological insulator. Phys. Rev. Lett. 100, 096407 (2008).

5. Fu, L. \& Kane, C. L. Josephson current and noise at a superconductor/ quantum-spin-Hall-insulator/superconductor junction. Phys. Rev. B 79, 161408 (2009)

6. Nayak, C. et al. Non-abelian anyons and topological quantum computation. Rev. Mod. Phys. 80, 1083-1159 (2008).

7. Konig, M. et al. The quantum spin Hall effect: Theory and experiment. J. Phys. Soc. Jpn 77, 031007 (2008)

8. Moore, G. \& Read, N. Nonabelions in the fractional quantum Hall effect. Nucl. Phys. B 360, 362-396 (1991).

9. Read, N. \& Green, D. Paired states of fermions in two dimensions with breaking of parity and time-reversal symmetries and the fractional quantum Hall effect. Phys. Rev. B 61, 10267-10297 (2000).

10. Sau, J. D., Lutchyn, R. M., Tewari, S. \& Sarma, S. D. Generic new platform for topological quantum computation using semiconductor heterostructures. Phys. Rev. Lett. 104, 040502 (2010).

11. Alicea, J. Majorana fermions in a tunable semiconductor device. Phys. Rev. B 81, 125318 (2010).

12. Kitaev, A. Y. Unpaired majorana fermions in quantum wires. Phys.-Usp. 44, 131-136 (2001)

13. Mourik, V. et al. Signatures of majorana fermions in hybrid superconductor-semiconductor nanowire devices. Science 336, 1003-1007 (2012)

14. Das, A. et al. Zero-bias peaks and splitting in Al-InAs nanowire topological superconductor as a signature of majorana fermions. Nature Phys. 8, $887-895$ (2012)

15. Rokhinson, L. P., Liu, X. \& Furdyna, J. K. The fractional a.c. Josephson effect in a semiconductor-superconductor nanowire as a signature of majorana particles. Nature Phys. 8, 795-799 (2012).

16. Lee, E. J. H. et al. Zero-bias anomaly in a nanowire quantum dot coupled to superconductors. Phys. Rev. Lett. 109, 186802 (2012).

17. Churchill, H. O. H. et al. Superconductor-nanowire devices from tunneling to the multichannel regime: Zero-bias oscillations and magnetoconductance crossover. Phys. Rev. B 87, 241401(R) (2013).

18. Finck, A. D. K. et al. Anomalous modulation of a zero-bias peak in a hybrid nanowire-superconductor device. Phys. Rev. Lett. 110, 126406 (2013)

19. Knez, I., Du, R-R. \& Sullivan, G. Andreev reflection of helical edge modes in InAs/GaSb quantum spin Hall insulator. Phys. Rev. Lett. 109, 186603 (2012).

20. Bernevig, B. A. \& Zhang, S-C. Quantum spin Hall effect. Phys. Rev. Lett. 96, 106802 (2006)

21. Bernevig, B. A., Zhang, S-C. \& Hughes, T. L. Quantum spin Hall effect and topological phase transition in HgTe quantum wells. Science 314, 1757-1761 (2006)

22. Konig, M. et al. Quantum spin Hall insulator state in HgTe quantum wells. Science 318, 766-770 (2007).

23. Roth, A. et al. Nonlocal transport in the quantum spin Hall state. Science 325, 294-297 (2009).

24. Schmidt, T. L., Rachel, S., Oppen, F. v. \& Glazman, L. I. Inelastic electron backscattering in a generic helical edge channel. Phys. Rev. Lett. 108, 156402 (2012)

25. Nowack, K. C. et al. Imaging currents in HgTe quantum wells in the quantum spin Hall regime. Nature Mater. 12, 787-791 (2013).

26. Tinkham, M. Introduction to Superconductivity (Dover Publications Inc., 2004).

27. Dynes, R. C. \& Fulton, T. A. Supercurrent density distribution in Josephson junctions. Phys. Rev. B 3, 3015-3023 (1971)

28. Oostinga, J. B. et al. Josephson supercurrent through the topological surface states of strained bulk HgTe. Phys. Rev. X 3, 021007 (2013). 
29. Sau, J. D. et al. Non-abelian quantum order in spin-orbit coupled semiconductors: Search for topological majorana particles in solid-state systems. Phys. Rev. B 82, 214509 (2010).

30. Jiang, L. et al. Unconventional Josephson signatures of majorana bound states. Phys. Rev. Lett. 107, 236401 (2011).

31. Mi, S. et al. Proposal for the detection and braiding of majorana fermions in a quantum spin Hall insulator. Phys. Rev. B 87, 241405(R) (2013).

\section{Acknowledgements}

We acknowledge A. Akhmerov and J. D. Sau for theoretical discussions. We acknowledge financial support from Microsoft Corporation Project Q, the NSF DMR-1206016, the STC Center for Integrated Quantum Materials, NSF Grant No. DMR-1231319, the DOE SCGF Program, the German Research Foundation (DFG-JST joint research programme 'Topological Electronics'), and EU ERC-AG programme (project 3-TOP).

\section{Author contributions}

All authors contributed collaboratively to all aspects of the work. Device fabrication measurements, data analysis and manuscript preparation were carried out at Harvard by S.H., H.R., T.W. and A.Y. All authors contributed to development of the fabrication process. P.L., M.M., C.B., H.B. and L.W.M. carried out the molecular beam epitaxy at Würzburg.

\section{Additional information}

Supplementary information is available in the online version of the paper. Reprints and permissions information is available online at www.nature.com/reprints.

Correspondence and requests for materials should be addressed to A.Y.

\section{Competing financial interests}

The authors declare no competing financial interests. 